\title{
Tumor growth model identification and analysis in case of C38 colon adenocarcinoma and B16 melanoma
}

\author{
Johanna Sápi*, Dániel András Drexler ${ }^{\dagger}$, István Harmati ${ }^{\dagger}$, Annamária Szeles $^{\dagger}$, Bernadett Kiss ${ }^{\dagger}$, \\ Zoltán Sápi ${ }^{\ddagger}$ and Levente Kovács* \\ * Óbuda University, John von Neumann Faculty of Informatics, IR Institute, \\ Bécsi út 96/B H-1034 Budapest, Hungary \\ Email: sapi.johanna@phd.uni-obuda.hu,kovacs.levente@nik.uni-obuda.hu \\ ${ }^{\dagger}$ Budapest University of Technology and Economics, Department of Control Engineering and Information Technology \\ Magyar tudósok krt 2. H-1117 Budapest, Hungary \\ Email: drexler@iit.bme.hu, harmati@iit.bme.hu, szeles.annam@gmail.com \\ ${ }^{\ddagger}$ Semmelweis University, 1st Department of Pathology and Experimental Cancer Research, \\ Üllői út 26. H-1085 Budapest, Hungary \\ Email: sapi.zoltan.dr@gmail.com
}

\begin{abstract}
Cancer fighting treatments are expanding, and a promising type, targeted molecular therapies have a new approach. The aim of these therapies is not to eliminate the whole tumor, but to control the tumor into a given state and keep it there. Explicit knowledge of tumor growth dynamics and the effects of targeted molecular therapies is crucial in tumor treatment development. We show the results of mouse experiments where tumor growth was investigated in case of C38 colon adenocarcinoma and B16 melanoma. Several curves were fitted and tumor growth dynamics was examined. Three attributes of tumor were measured: tumor volume, tumor mass and vascularization; and tumor growth dynamics was examined. Tumor volume was measured with digital caliper, vascularization was investigated with CD31 antibody immunohistochemistry staining on frozen sections. The relationship between these tumor attributes were examined with linear regression analysis. The dynamics of tumor growth was identified as a second order linear system.
\end{abstract}

\section{INTRODUCTION}

Conventional cancer therapies are surgical oncology, chemotherapy and radiotherapy. With surgical oncology [1] the tumor cells can be totally removed (zero-order kinetics); in contrast using chemotherapy [2] or radiation therapy [3], only a fraction of tumor cells are killed (first-order kinetics). The problem with surgical intervention is the fact, that tumor can recurrent in many cases [4]. Chemotherapy and radiotherapy act in general ways (by killing rapidly dividing cells), so these treatments have a lot of side effects and tumor cells can become resistant to chemotherapy drugs, which makes the usage of new drugs necessary. That is the reason, why there is a new, dynamically developing therapeutic group called targeted molecular therapies (TMTs). These treatments fight specifically against different cancer mechanisms, hereby these therapies can be more effective and have limited side effects.

The main differences between conventional cancer therapies and targeted molecular therapies are not only the acting ways, but also the goals. Using conventional treatments, there is no need to know how cancer cells are developing and which mechanisms are used to circumvent the immune system. The goal of conventional cancer therapies is to eliminate the tumor mass, but after a certain time the tumor can recrudesce and give metastasis. Targeted therapy represents a new approach: it acts in specific molecular ways, and the goal is to prevent tumor cells from growth and develop; hence preventing toxicity. This is more important than eliminating the tumor mass - for the patients there is a better chance of survival if they have inactive tumor mass, than if they don't have tumor for a while, but there is the risk of recurrence. In order to develop targeted therapies, it is required to analyze tumor growth and explore causal factors, but with this knowledge these therapies have led to truly tailored therapy [5] with reduced side effects [6].

The process of analyzing tumor growth belongs to the science of molecular oncology. The aim of targeted molecular therapies is not to eliminate the whole tumor, but to control the tumor into a given state and keep it there. This task belongs to the science of control engineering that can provide effective assistance to model-based therapies, which examine tumor growth as a dynamic system [7], [8]. We have investigated existing tumor growth models from the literature and designed different control algorithms [9], [10], [11], [12]. In this paper we summarize the results of our animal experiments and present our identified tumor growth model for C38 colon adenocarcinoma and B16 melanoma.

The paper is organized as follows. In Section II, the oncopathological background (properties of the investigated tumor types) of the research is described. In Section III, the methods used in the present study are described. Experimental results are shown is Section IV, followed by the identification of tumor growth dynamics and correlation analysis of tumor 
attributes. The paper ends in Section V with the conclusion and further research possibilities.

\section{ONCOPATHOLOGICAL BACKGROUND OF THE EXPERIMENT}

C38 colon adenocarcinoma is a mouse tumor, which is originated from columnar epithelium of colon's mucosa. Adenocarcinoma means "a malignant epithelial tumor with glandular differentiation or mucin production" [13]. Properties of C38 adenocarcinoma are: (a) because this is a mouse tumor, it grows fast in mice (since 2-3 weeks it reaches a lethal size); (b) also because its specificity, there is no need to use immunosuppressed mice; (c) a piece of tumor can be implanted subcutaneously into the mice; (d) typically does not metastasize; (e) tumor cells inflict strong hypoxial reaction [14]. C38 tumor also has a large relative vascular area [15], which is beneficial if we would like to examine the effect of angiogenic inhibition later.

B16 melanoma ([16], [17]) is a mouse tumor, it is a primer melanoma. "Melanomas are malignant tumors derived from melanocytes" [13]. The pattern can be variable, usually the cytoplasm contains melanin granules. Properties of B16 melanoma are: (a) because this is a mouse tumor, it grows very fast in mice (since 2 weeks it reaches a lethal size); (b) also because its specificity, there is no need to use immunosuppressed mice; (c) it can be injected intramuscularly; (d) typically does not metastasize. B16 mice melanoma can be used as a model for human melanoma [18].

C38 colon adenocarcinoma and B16 melanoma are widely and for a long while used mouse experimental tumors from the standard NCI (National Cancer Institute) screening [19], [20].

\section{DESCRIPTION OF THE EXPERIMENT}

In this section we give a brief description of the methods used during the mouse experiments.

\section{A. Tumor implantation}

In case of C38 colon adenocarcinoma a piece of tumor was transplanted subcutaneously in the recipient animal. In case of B16 melanoma $2 \cdot 10^{6}$ tumor cells were injected intramuscularly for a mouse.

\section{B. Tumor volume measurement}

The first measurement after tumor implantation occured when the tumors have reached an average volume of $50-$ $60 \mathrm{~mm}^{3}$ [21]. It was the $5 \mathrm{th}$ day for $\mathrm{C} 38$ colon adenocarcinoma, and the $10 t h$ day for B16 melanoma. Tumor diameters (width, length) were measured with a digital caliper, the third dimension of the tumor was approximated with the arithmetic mean of its width and length, multiplied by $2 / 3$. Thus, the tumor volume in $\mathrm{mm}^{3}$ was calculated by the formula:

$$
\text { Volume }=\text { width } \cdot \text { length } \cdot \frac{\text { width }+ \text { length }}{2}
$$

\section{Sacrificing mice}

Mice were sacrificed when the tumor reached a lethal size. It was the 24th day for C38 colon adenocarcinoma, and the 19th day for B16 melanoma. After sacrificing the mice, the tumors were removed, and their volume and mass was measured. Then, tumors were cut into two pieces: one piece was stored in formalin, and the other piece was freezed using liquid nitrogen (with temperature of $-200^{\circ} \mathrm{C}$ ).

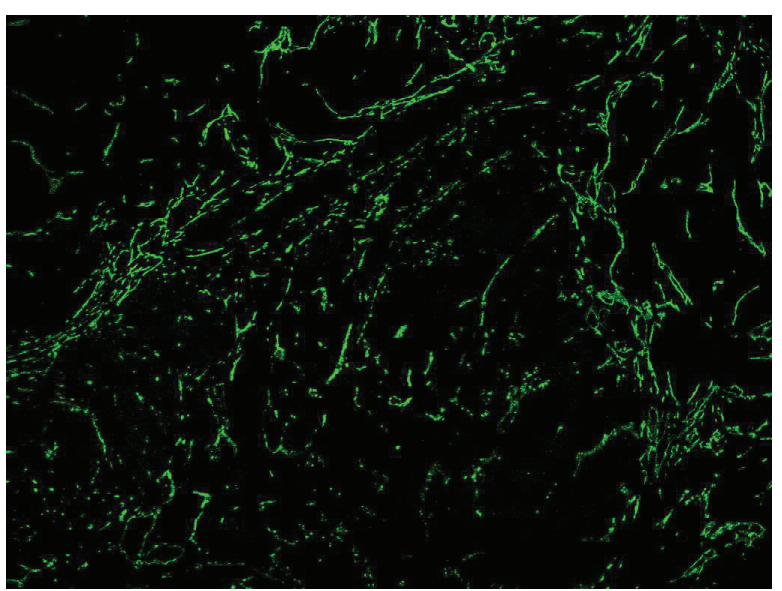

Fig. 1. Investigation of C38 colon adenocarcinoma's vascularization: CD31 antibody (green, fluorescent) immunohistochemistry staining on frozen sections

\section{Tumor sample processing}

Tumor morphology can be investigated using standard Haematoxylin Eosin (H\&E) staining [22] with the samples stored in formalin. The freezed samples were used to create frozen sections [23] of the tumor. Tumor vasculation can be stained in vitro using CD31 antibody immunohistochemistry staining [24] on frozen sections. After staining, fluorescence pictures were done with confocal microscope (Fig. 1). From these pictures vascularization area was calculated by ImageJ [25] software.

\section{ANALYSIS OF EXPERIMENTAL RESULTS}

In this section we analyze the measurement data acquried from the animal experiments, and carry out parametric identification for the tumor growth dynamics supposing that tumor growth is described by a second order linear system.

\section{A. Experimental data}

At the beginning of the experiment 12 mice were implanted with C38 colon adenocarcinoma. One of them died in the $18 \mathrm{th}$ day, and another one in the 23th day. Thus 10 mice were sacrificed at the $24 t h$ day of the experiment.

B16 melanoma was implanted into 11 mice. One mouse died at the $18 t h$ day, therefore 10 mice were sacrificed at the $19 t h$ day of the experiment. 


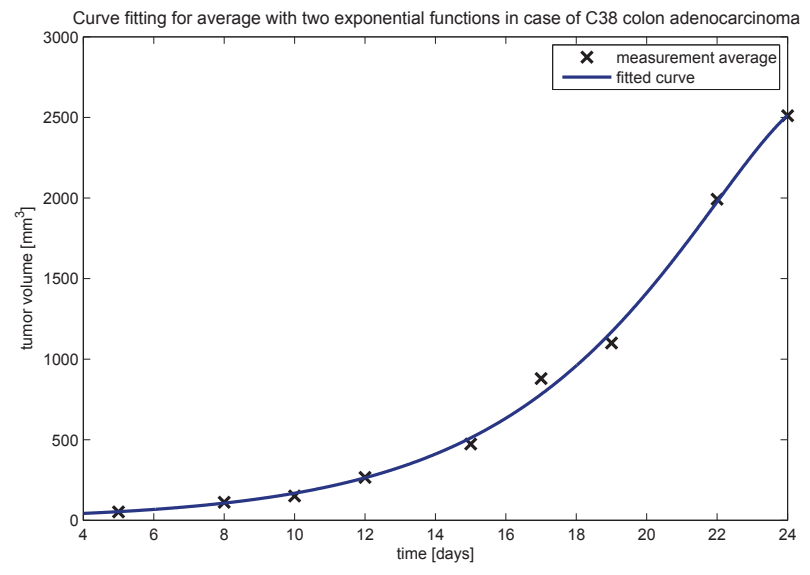

Fig. 2. Exponential curve fitting for average in case of $\mathrm{C} 38$ colon adenocarcinoma $(y(t)=-0.076 \cdot \exp (0.4239 t)+16.87 \cdot \exp (0.2329 t)$

\section{B. Results of C38 colon adenocarcinoma experiment}

Based on the tumor volume measurements, our goal is to identify the model of tumor growth dynamics. From our results the general assumption that tumor cells grow exponentially [26] is verifiable. The simplest dynamic model is a linear one, thus the response of the system consists of expontential functions. Since our aim is to develop a model for antiangiogenic therapy, we want to identify the dynamics behind tumor growth and vasculature growth, thus we are seeking for a second order system. The second order system has two exponential functions in its response, thus parametric identification was carried out by fitting a curve with two exponential functions. The result of curve fitting on the average of the measurents is in Fig. 2. The response of the system is described by:

$$
y(t)=-0.076 \cdot \exp (0.4239 t)+16.87 \cdot \exp (0.2329 t)
$$

The time constants of the identified model are $T_{1}=2.3589$ days, and $T_{2}=4.2938$ days. The coefficients of the exponentials are positive, thus the system is unstable, as it is required from a tumor growth.

This fitting were done with Matlab and results were verified with SPSS [27]. Using SPSS, we have examined other fitting curve types and four curves had equal or better coefficient of determination than 0.99 (exponential $R^{2}=0.990$, growth $R^{2}=0.990$, compound $R^{2}=0.990$, cubic $R^{2}=0.991$ ). Cubic curve fits better for the sample points than the other, but outside of the sample range, the extrapolation is worse. Exponential, growth and compound models fitted the same curves.

Another model used to describe tumor growth is Gompertz curve [28], which described a dynamic process that has a plateu. In this model tumor cell number also depends on the initial tumor size, the elapsed time and a constant, but at the end of the growth period growth narrows and cell number has a plateau:

$$
N(t)=N_{0} \cdot \exp \left[\ln \left(\frac{N_{\infty}}{N_{0}}[1-\exp (-b t)]\right)\right],
$$

where $N_{\infty}$ is the plateau and parameter $b$ is related to the initial tumor growth rate. This model describes that tumor growth is nutrient-, and oxygen-limited. However, in the plateau phase tumor size and toxicity is lethal for the host organism without therapy. In our experiments the last measured tumor volume is approximately equal to the plateau cell number. Nevertheless Gompertz curve is more difficult than the exponential one, and may even vary considerably for patients with the same type of cancer.

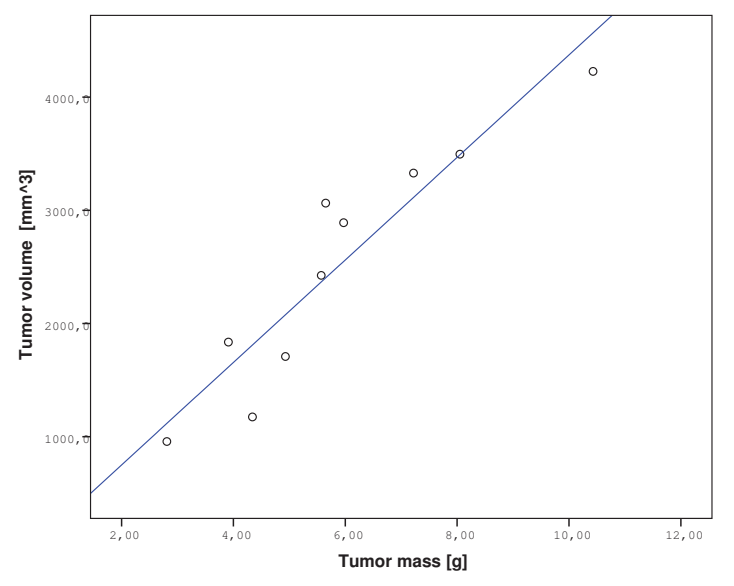

Fig. 3. Linear regression between tumor mass and volume in case of C38 colon adenocarcinoma $\left(R^{2}=0.871, R=0.933, p<0.0001\right)$

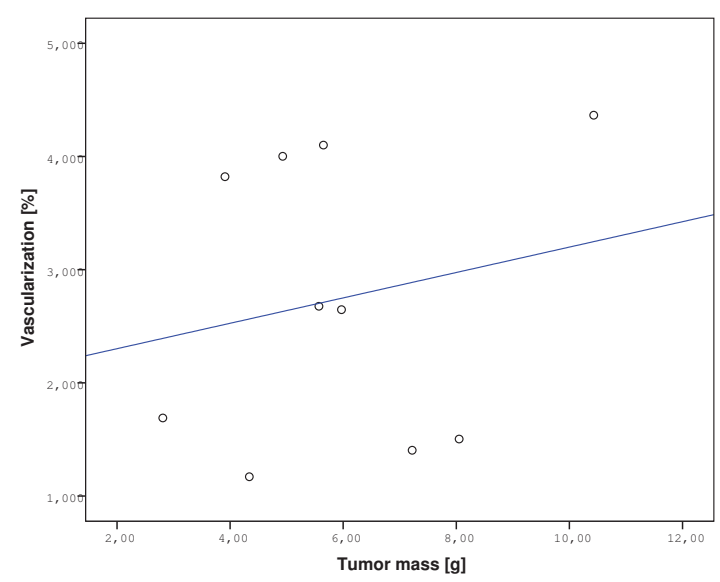

Fig. 4. Linear regression between tumor mass and vascularization in case of C38 colon adenocarcinoma $\left(R^{2}=0.039, R=0.198, p=0.584\right)$

As discussed previously, we have measured three data of the lethal sized tumor: tumor volume, tumor mass and vascularization. We have investigated the relationship between these tumor attributes with linear regression analysis [29]. In Fig. 3 the relationship between tumor mass and volume can be seen. 


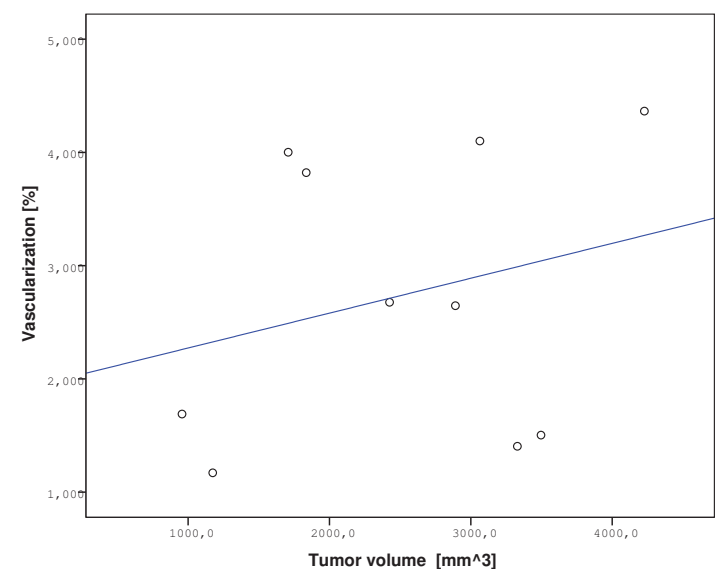

Fig. 5. Linear regression between tumor volume and vascularization in case of C38 colon adenocarcinoma $\left(R^{2}=0.069, R=0.263, p=0.462\right)$

The coefficient of determination is $R^{2}=0.871$, this means that $87.1 \%$ variability in a data can be explained by the given statistical model. Pearson correlation coefficient is $R=0.933$, thus there is a strong correlation (linear dependence) between these variables. Using Analysis of Variance (ANOVA) test [30] we can decide that the regression analysis is valid or not. In this case a strong significant regression relationship can be detected $(p<0.0001)$. C38 colon adenocarcinoma is a solid tumor - this type of tumor usually does not contain cysts or liquid area, thus tumor mass has high density.

The association between tumor mass and vascularization is shown in Fig. 4. The coefficient of determination is $R^{2}=$ 0.039 , Pearson correlation coefficient is $R=0.198$, and ANOVA $\mathrm{p}$-value is $p=0.584$. From each parameter we can see that there is a weak, not significant relationship between tumor mass and vascularization. We get similar results in case of tumor volume and vascularization (Fig. 5). The coefficient of determination is $R^{2}=0.069$, Pearson correlation coefficient is $R=0.263$, and ANOVA p-value is $p=0.462$. This can be explained by the following. Rapidly dividing tumor cells need lots of oxygen. When proliferation begins, small sized tumor can pick up oxygen from near capillaries. After a certain size (1-2 $\mathrm{mm}$ diameter) tumor development stops, because a part of the tumor gets too far from capillaries and can't pick up enough oxygen. Tumor needs own blood vessels to grow, the process of forming new blood vessels is called angiogenesis [31], [32]. However, due to this hurried vessel forming, a part of tumor still can't get enough oxygen, whereupon these cells first inflict hypoxial reaction, then die. In C38 colon adenocarcinoma there are several necrotic regions, thus the whole mass cointains relatively few viable cells and vessels [33].

\section{Results of B16 melanoma experiment}

In case of B16 melanoma, we were also seeking for a linear second order system to describe the tumor growth dynamics. The average values of the measurements and the fitted multiexponential curve is in Fig. 6. The result of the parametric identification is the following function:

$$
y(t)=-511.6 \cdot \exp (0.54781 t)+512.3 \cdot \exp (0.54775 t)
$$

The time constants of the system are $T_{1}=1.8256$ days and $T_{2}=1.8254$ days. The parametric idenfitication results in almost identical time constants, however the multiexponential characteristics is important. This simple model even results in a platue like characteristics at high tumor volume values (Fig. 6.), without the nonlinear model of the Gompertzian growth. The coefficients of the exponential functions are positive in this case as well, resulting in an unstable system.

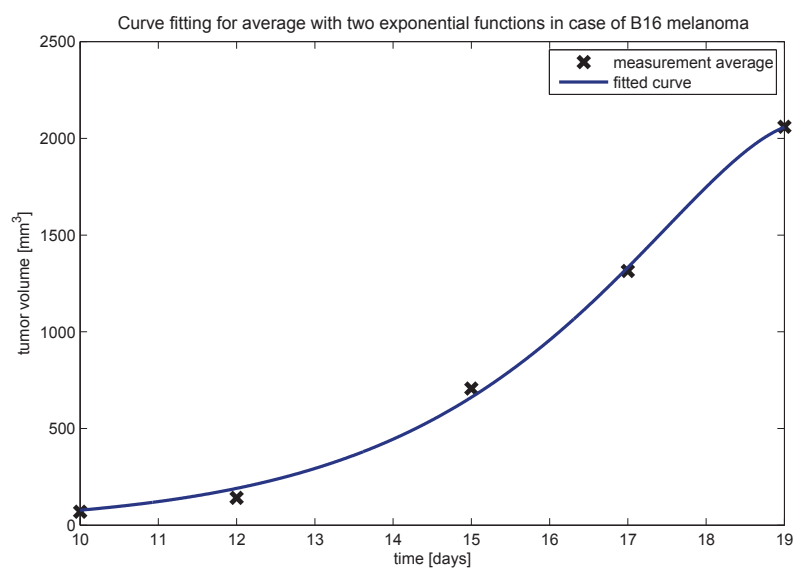

Fig. 6. Exponential curve fitting for average in case of B16 melanoma $(y(t)=-511.6 \cdot \exp (0.54781 t)+512.3 \cdot \exp (0.54775 t)$

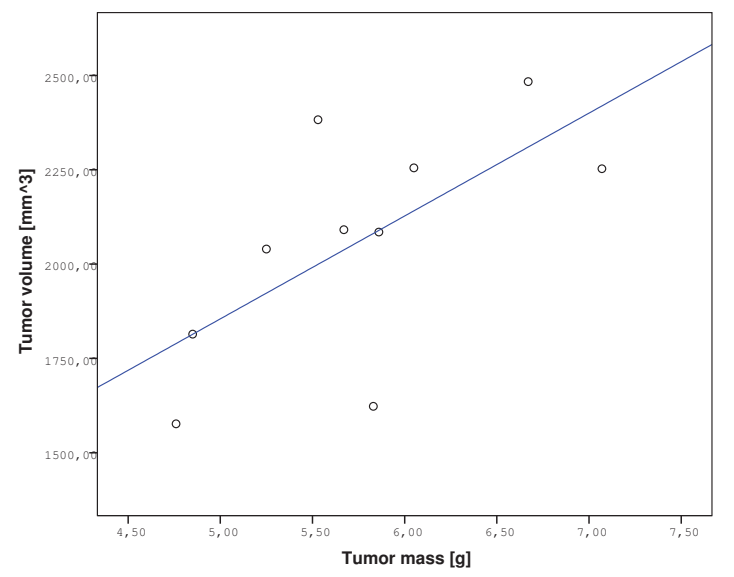

Fig. 7. Linear regression between tumor mass and volume in case of B16 melanoma $\left(R^{2}=0.421, R=0.649, p=0.042\right)$

As at analysis of C38 colon adenocarcinoma, this fitting was also done with Matlab and results were verified with SPSS. Examined best fitting curve types and coefficient of determination values are: exponential $R^{2}=0.955$, growth $R^{2}=0.955$, compound $R^{2}=0.955$, cubic $R^{2}=0.981$. 
Also in this case cubic fits better, but has the same problem (wrong extrapolation and prediction). Exponential, growth and compound models fitted the same curves.

In Fig. 7 the relationship between tumor mass and volume can be seen. The coefficient of determination is $R^{2}=0.421$, Pearson correlation coefficient is $R=0.649$. These parameters show correlation, but not as strong as in case of C38 colon adenocarcinoma. Using ANOVA test, also a weaker, but significant regression relationship can be detected $(p=0.042)$. B16 melanoma is a solid tumor as well, but cell growth leads to necrosis and liquefaction of muscle tissues. Because of that the removed mass contains liquefied areas, which have lower density.

The association between tumor mass and vascularization is shown in Fig. 8. The coefficient of determination is $R^{2}=$ 0.215 , Pearson correlation coefficient is $R=0.463$, and ANOVA p-value is $p=0.177$. The relationship between tumor volume and vascularization can be seen in Fig. 9. The coefficient of determination is $R^{2}=0.029$, Pearson correlation coefficient is $R=0.170$, and ANOVA p-value is $p=0.638$. Vascularization does not have significant relationship with tumor mass, neither with tumor volume.

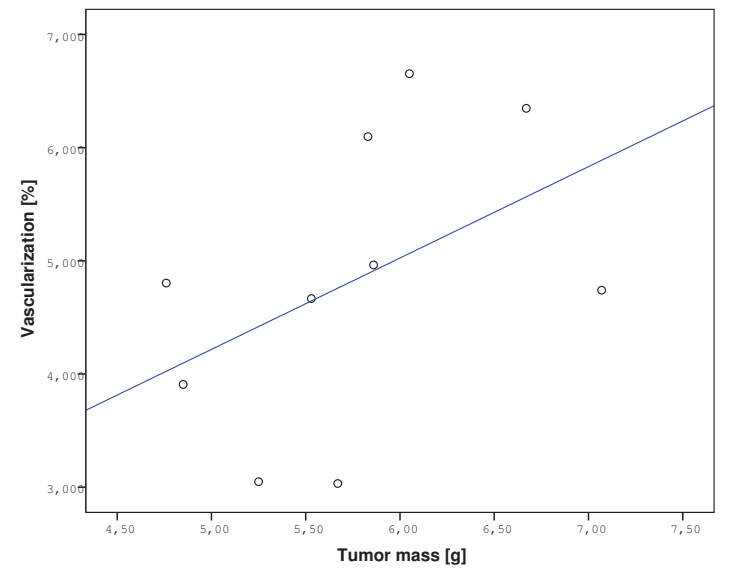

Fig. 8. Linear regression between tumor mass and vascularization in case of B16 melanoma $\left(R^{2}=0.215, R=0.463, p=0.177\right)$

\section{CONCLUSION}

Our results show that tumor growth dynamics can be described with a second order linear system. Examining the tumor attributes, we can say that not each attributes correlates, thus not only tumor mass and tumor volume is important to measure. The relevant tumor attribute, that have to be measured is based on the therapy applied. In case of antiangiogenic therapy, vascularization can be more important than tumor mass or tumor volume. Further work will be contain other mouse experiments, where we will investigate tumor growth under antiangiogenic therapy. Moreover, other modelling aspects will be investigated similar to [34], [35], [36].

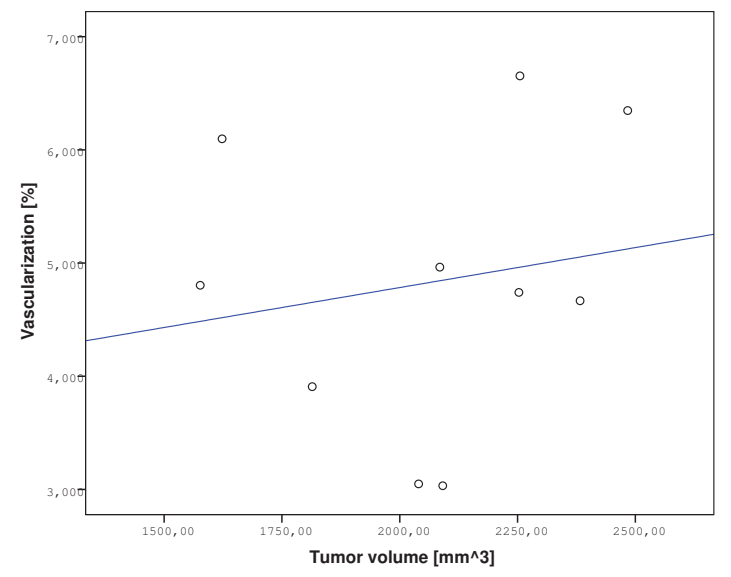

Fig. 9. Linear regression between tumor volume and vascularization in case of B16 melanoma $\left(R^{2}=0.029, R=0.170, p=0.638\right)$

\section{ACKNOWLEDGMENT}

The authors say special thanks to Katalin Dezsô MD. $\mathrm{PhD}$ from the 1st Department of Pathology and Experimental Cancer Research Institute from Semmelweis University, Budapest, for making the fluorescence pictures with confocal microscope. The authors also say thanks to András Sztodola, from the 1st Department of Pathology and Experimental Cancer Research Institute from Semmelweis University Animal Laboratory, for his technical help during the experiments. Levente Kovács was supported by the János Bolyai Research Scholarship of the Hungarian Academy of Sciences. Annamária Szeles is supported in the frames of TÁMOP 4.2.4.A/1-111-2012-0001 "National Excellence Program - Elaborating and operating an inland student and researcher personal support system". The project was subsidized by the European Union and co-financed by the European Social Fund.

\section{REFERENCES}

[1] R. E. Pollock, Advanced Therapy in Surgical Oncology. BC Decker, Hamilton, Ontario, Canada, 2008.

2] R. Page and C. Takimoto, "Principles of chemotherapy, Cancer management: a multidisciplinary approach," http://www.thymic.org/uploads/ reference_sub/03chemoprinc.pdf/, 2001, 01.02.2013.

[3] M. J. Gazda and R. C. Lawrence, "Principles of radiation therapy, Cancer management: a multidisciplinary approach," http://www.thymic. org/uploads/reference_sub/02radtherapy.pdf/, 2001, 01.02.2013.

[4] A. Tavare, N. Perry, L. Benzonana, M. Takata, and D. Ma, "Cancer recurrence after surgery: direct and indirect effects of anesthetic agents," Int J Cancer, vol. 130(6), pp. 1237-1250, 2012.

[5] D. E. Gerber, "Targeted therapies: a new generation of cancer treatments," Am Fam Physician., vol. 77(3), pp. 311-319, 2008.

[6] H. H. Kreipe and R. Wasielewski, "Beyond typing and granding: Target analysis in individualized therapy as a new challenge for tumour pathology," in Recent Results In Cancer Research, Targeted Therapies in Cancer, M. Dietel, Ed. Springer - Verlag Berlin Heidelberg, 2007.

[7] L. Kovács, P. Szalay, T. Ferenci, D. A. Drexler, J. Sápi, I. Harmati, and Z. Benyó, "Modeling and optimal control strategies of diseases with high public health impact," INES 2011 - 15th International Conference on Intelligent Engineering System (IEEE), pp. 23-28, June 2011, Poprad, Slovakia. 
[8] L. Kovács, P. Szalay, T. Ferenci, J. Sápi, P. I. Sas, D. A. Drexler, I. Harmati, B. Benyó, and A. Kovács, "Modeling and optimal control strategies of diseases with high public health impact," INES 2012 - 16th International Conference on Intelligent Engineering Systems (IEEE), pp. 531-536, June 2012, Lisbon, Portugal.

[9] D. A. Drexler, L. Kovács, J. Sápi, I. Harmati, and Z. Benyó, "Modelbased analysis and synthesis of tumor growth under angiogenic inhibition: a case study." IFAC WC 2011 - 18th World Congress of the International Federation of Automatic Control, pp. 3753-3758, August 2011, Milano, Italy.

[10] D. A. Drexler, J. Sápi, A. Szeles, I. Harmati, A. Kovács, and L. Kovács, "Flat control of tumor growth with angiogenic inhibition," SACI 2012 - 6th International Symposium on Applied Computational Intelligence and Informatics (IEEE), pp. 179-183, May 2012, Timisoara, Romania.

[11] J. Sápi, D. A. Drexler, I. Harmati, Z. Sápi, and L. Kovács, "Linear statefeedback control synthesis of tumor growth control in antiangiogenic therapy," SAMI 2012 - 10th International Symposium on Applied Machine Intelligence and Informatics (IEEE), pp. 143-148, January 2012, Herlany, Slovakia.

[12] A. Szeles, J. Sápi, D. A. Drexler, I. Harmati, Z. Sápi, and L. Kovács, "Model-based angiogenic inhibition of tumor growth using modern robust control method," IFAC BMS 2012 - 8th IFAC Symposium on Biological and Medical Systems, pp. 113-118., 2012, Budapest: IFAC by Pergamon Press.

[13] W. D. Travis et al., Pathology and Genetics: Tumours of the Lung, Pleura, Thymus and Heart (World Health Organization Classification of Tumours). International Agency for Research on Cancer, 2004.

[14] A. S. Ljungkvist, J. Bussink, J. H. Kaanders et al., "Hypoxic cell turnover in different solid tumor lines," Int J Radiat Oncol Biol Phys., vol. 62(4), pp. 1157-1168, 2005

[15] H. W. van Laarhoven, G. Gambarota, J. Lok et al., "Carbogen breathing differentially enhances blood plasma volume and 5-fluorouracil uptake in two murine colon tumor models with a distinct vascular structure,' Neoplasia., vol. 8(6), pp. 477-487, 2006.

[16] Abcam, "B16 (mouse melanoma cell line) nuclear lysate," http://www. abcam.com/B16-Mouse-melanoma-cell-line-Nuclear-Lysate-ab14638. html, 01.02.2013.

[17] ATCC, "B16-F10 product description," http://www.atcc.org/ ATCCAdvancedCatalogSearch/ProductDetails/tabid/452/Default.aspx? ATCCNum=CRL-6475\&Template $=$ cellBiology, 01.02.2013.

[18] W. W. Overwijk and N. P. Restifo, "B16 as a mouse model for human melanoma," Curr Protoc Immunol., vol. Chapter 20., p. Unit 20., 2001.

[19] M. H. Silbermann, B. Vecht, G. Stoter, K. Nooter, and J. Verweij, "Combination therapy of ACNU and ifosfamide in tumor bearing mice with M2661 breast cancer, B16 malignant melanoma or C38 colon cancer," Eur J Cancer., vol. 26(3), pp. 321-325, 1990.

[20] N. C. Institute, "eMICE: electronic Models Information, Communication, and Education," http://emice.nci.nih.gov/, 01.02.2013.

[21] P. Online, "Xenograft tumor model protocol," http://www.protocol-online.org/prot/Protocols/ Xenograft-Tumor-Model-Protocol-3810.html, 01.02.2013.

[22] A. H. Fischer, K. A. Jacobson, J. Rose, and R. Zeller, "Hematoxylin and eosin staining of tissue and cell sections," CSH Protoc., vol. pdb.prot4986, 2008.

[23] C. S. Technology, "Immunohistochemistry frozen section protocol," http //www.cellsignal.com/support/protocols/4060_ihcf.html, 01.02.2013.

[24] I. WORLD, "CD31 antibody staining protocol for immunohistochemistry," http://www.ihcworld.com/_protocols/antibody_protocols/ cd31_pharmingen.htm, 01.02.2013.

[25] ImageJ, "Image processing and analysis in Java," http://rsbweb.nih.gov/ ij/index.html, 01.02.2013.

[26] S. E. Shackney, "Tumor growth, cell cycle kinetics, and cancer treatment," in Medical Oncology: Basic Principles and Clinical Management of Cancer, P. Calabresi and P. S. Schein, Eds. McGraw Hill, New York, 1993.

[27] SPSS, "SPSS software: Predictive analytics software and solutions," http://www-01.ibm.com/software/analytics/spss/, 01.02.2013.

[28] E. D. Yorke, Z. Fuks, L. Norton, W. Whitmore, and C. C. Ling, "Modeling the development of metastases from primary and locally recurrent tumors: comparison with a clinical data base for prostatic cancer," Cancer Res., vol. 53(13), pp. 2987-2993, 1993.

[29] D. C. Montgomery, E. A. Peck, and G. G. Vining, Introduction to Linear Regression Analysis, fifth edition. John Wiley \& Sons, Inc., Hoboken, New Jersey, 2012.
[30] M. G. Larson, "Statistical primer for cardiovascular research. analysis of variance," Circulation, vol. 117, pp. 115-121, 2008.

[31] A. Hoeben, B. Landuyt, M. Highley, H. Wildiers, A. T. Van Oosterom, and E. A. De Bruijn, "Vascular endothelial growth factor and angiogenesis," Pharmacol Rev., vol. 56, pp. 549-580, 2004.

[32] G. Bergers and L. E. Benjamin, "Tumorigenesis and the angiogenic switch," Nat Rev Cancer, vol. 3(6), pp. 401-410, 2003.

[33] Y. J. Kamm, A. Heerschap, G. Rosenbusch, I. M. Rietjens, J. Vervoort, and D. J. Wagener, "5-fluorouracil metabolite patterns in viable and necrotic tumor areas of murine colon carcinoma determined by $19 \mathrm{f} \mathrm{nmr}$ spectroscopy," Magn Reson Med., vol. 53(13), pp. 2987-2993, 1996.

[34] A. Rodić and G. Mester, "The modeling and simulation of an autonomous quad-rotor microcopter in a virtual outdoor scenario," Acta Polytechnica Hungarica, vol. 8(4), pp. 107-122, 2011.

[35] M. Badida, R. Králiková, and E. Lumnitzer, "Modeling and the use of simulation methods for the design of lighting systems," Acta Polytechnica Hungarica, vol. 8(2), pp. 91-102, 2011.

[36] F. Toorani and H. Farahmandzad, "Gray-box modeling of a pneumatic servo-valve," Acta Polytechnica Hungarica, vol. 7(5), pp. 129-142, 2010. 